\title{
Saúde mental da criança em tempos de pandemia da COVID-19: revisão integrativa
}

\section{Children's mental health in times of a COVID-19 pandemic: integrative review}

Salud mental infantil en tiempos de pandemia de COVID-19: revisión integrativa

Brenda Kelly Pontes Soares

ORCID: https://orcid.org/0000-0001-7873-1653 Universidade Federal do Rio Grande do Norte, Brasil

E-mail: brendaa.pontes@gmail.com

Roseane Morais da Silva

ORCID: https://orcid.org/0000-0003-4260-5553

Universidade Federal do Rio Grande do Norte, Brasil

E-mail: roseane.morais@outlook.com.br

Rayssa da Silva Felinto

ORCID: https://orcid.org/0000-0003-0191-5539

Universidade Federal do Rio Grande do Norte, Brasil

E-mail: rayssafelinto@outlook.com

Albenize de Azevêdo Soares

ORCID: https://orcid.org/0000-0002-3008-0043

Universidade Federal do Rio Grande do Norte, Brasil

E-mail: nize.azevedo@hotmail.com

Jayara Mikarla de Lira

ORCID: https://orcid.org/0000-0002-1707-0983

Universidade Federal do Rio Grande do Norte, Brasil

E-mail: jayara-mikarla@ hotmail.com

Flaviane Dantas de Araújo

ORCID: https://orcid.org/0000-0003-2142-7372

Universidade Federal do Rio Grande do Norte, Brasil

E-mail: flaviane.dantas.105@ufrn.edu.br

Lisandra Mikaely Barboza da Silva

ORCID: https://orcid.org/0000-0002-1862-7990

Universidade Federal do Rio Grande do Norte, Brasil

E-mail: lisbarboza@outlook.com

Ana Caroline da Fonseca Nunes

ORCID: https://orcid.org/0000-0002-8445-8873

Universidade Federal do Rio Grande do Norte, Brasil

E-mail: carolfonsecanunes2000@ gmail.com

Mayara Gabriela Cândido de Oliveira

ORCID: https://orcid.org/0000-0002-9669-5999

Universidade Federal do Rio Grande do Norte, Brasil

E-mail: mayaragb4@hotmail.com

Heleni Aires Clemente

ORCID: https://orcid.org/0000-0002-2180-6754

Universidade Federal do Rio Grande do Norte, Brasil

E-mail: heleni.aires11@hotmail.com

José Adailton da Silva

ORCID: https://orcid.org/0000-0002-6037-7649

Departamento de Saúde Coletiva, Brasil

E-mail: silva.adaiton@ufrn.edu.br

Ana Gabriella Costa Lemos da Silva

ORCID: https://orcid.org/0000-0001-7761-2708

Hospital Pediátrico Maria Alice Fernandes, Brasil

E-mail: gabriella_lemos_06@yahoo.com.br

\section{Resumo}

Objetivo: Realizar um levantamento na literatura científica dos impactos da COVID-19 na saúde mental de crianças. Metodologia: Trata-se de uma revisão integrativa realizada a partir de buscas nas bases científicas Scopus, PubMed, Embase e Medline. Foram incluídos artigos de pesquisas originais, open access e publicados entre 2020 a 2021. Para a chave de busca foram utilizados os descritores "Coronavirus Infections", "Child Health", Child Preschool" e "Mental Health" e sinonímias em inglês. Foram encontrados 280 estudos dos quais 6 foram incluídos. Resultados: Todos os estudos selecionados apontaram nível de evidência $\mathrm{V}$ e encontraram diferentes impactos negativos que a pandemia da COVID-19 causou na saúde mental de crianças, afetando suas ações, pensamentos e emoções. Conclusão: Diante das implicações causadas pela pandemia na saúde mental das crianças, é importante a adoção de 
medidas que possam mantê-las saudáveis, como a prática de atividades físicas dentro de suas próprias casas, brincadeiras que não envolvam a utilização de telas e a realização de refeições em família.

Palavras-chave: COVID-19; Isolamento social; Quarentena; Pandemias; Saúde mental; Saúde da criança.

\begin{abstract}
Objective: Conduct a survey in the scientific literature of the impacts of COVID-19 on children's mental health. Methodology: This is an integrative review carried out based on searches in the scientific databases Scopus, PubMed, Embase and Medline. Original research articles, open access and published between 2020 and 2021 were included. For the search key, the descriptors "Coronavirus Infections", "Child Health", "Child Preschool" and "Mental Health" and synonyms in English were used. 280 studies were found, 6 of which were included. Results: All selected studies indicated evidence level $\mathrm{V}$ and found different negative impacts that the COVID-19 pandemic caused on the mental health of children, affecting their actions, thoughts and emotions. Conclusion: In view of the implications caused by the pandemic on the mental health of children, it is important to adopt measures that can keep them healthy, such as the practice of physical activities inside their own homes, games that do not involve the use of screens and performance of family meals.
\end{abstract}

Keywords: COVID-19; Social isolation; Quarantine; Pandemics; Mental health; Child health.

\title{
Resumen
}

Objetivo: Realizar una encuesta en la literatura científica sobre los impactos del COVID-19 en la salud mental de los niños. Metodología: Se trata de una revisión integradora realizada a partir de búsquedas en las bases de datos científicas Scopus, PubMed, Embase y Medline. Se incluyeron artículos de investigación originales, de acceso abierto y publicados entre 2020 y 2021. Para la clave de búsqueda se utilizaron los descriptores "Infecciones por coronavirus", "Salud infantil", "Preescolar infantil" y "Salud mental" y sinónimos en inglés. Se encontraron 280 estudios, de los cuales se incluyeron 6. Resultados: Todos los estudios seleccionados indicaron nivel de evidencia V y encontraron diferentes impactos negativos que la pandemia COVID-19 provocó en la salud mental de los niños, afectando sus acciones, pensamientos y emociones. Conclusión: Ante las implicaciones que la pandemia tiene sobre la salud mental de los niños, es importante adoptar medidas que puedan mantenerlos saludables, como la práctica de actividades físicas dentro de sus propios hogares, juegos que no impliquen el uso de pantallas y realización de comidas familiares.

Palabras clave: COVID-19; Aislamiento social; Cuarentena; Pandemias; Salud mental; Salud del niño.

\section{Introdução}

A doença causada pelo novo coronavírus (COVID-19) resultou em muitas mortes, principalmente ocasionada pela Síndrome respiratória aguda grave (SARS). No final do ano de 2019, a cidade de Wuhan, na província de Hubei, China, apresentou um surto de insuficiência respiratória aguda de pessoas acometidas por um novo coronavírus, que acabou provocando mais de 800 mortes, infectando em torno de 70.000 pessoas nos primeiros 5 dias da epidemia e, desde então, ocorreu uma rápida disseminação mundial. A Organização Mundial de Saúde (OMS) declarou Emergência de Saúde Pública de Importância Internacional (ESPII) em 30 de janeiro de 2020 e pandemia em 11 de março de 2020 (Sant'Ana et al., 2020 ; Sousa, Sales, Rodrigues, Rocha, \& Oliveira, 2020; Campos et al., 2020; Wang, Horby, Hayden \& Gao, 2020; World Health Organization [WHO], 2020).

Em relação aos aspectos clínicos da doença, a COVID-19 pode apresentar diferentes níveis de gravidade. Nos casos mais leves, manifesta-se geralmente com sintomas iniciais parecidos com de outras doenças respiratórias e assim permanece até a recuperação do infectado. Já nos casos mais graves, pode evoluir para necessidade de uso de suporte ventilatório ou até mesmo o óbito. De forma geral, os sintomas mais comuns são febre, tosse seca, fadiga, mialgia, anosmia, hiposmia e ageusia, podendo também ocasionar dispneia ou Síndrome Respiratória Aguda Grave (Guan et al., 2020; Rocha, Veloso, Bezerra, Gomes, \& Marcolino, 2021; Wang et al., 2020).

Quanto ao público, os primeiros achados mostraram que as crianças com COVID 19 geralmente apresentavam infecções respiratórias com sintomas mais leves quando comparados com a situação clínica dos adultos. Para o grupo pediátrico, sintomas como febre, tosse seca, desconforto na garganta, congestão nasal, espirro e rinorreia eram os mais comuns (Gonçalves, Candeia, Andrade, Batista, \& Sousa, 2020).

Por outro lado, devido à rapidez com que se espalhou em diversos países do mundo, a pandemia da COVID-19 
resultou na implementação de medidas de isolamento social, como fechamento de escolas, distanciamento social e quarentena domiciliar. Com isso, crianças e adolescentes experimentaram um estado prolongado de isolamento físico de seus colegas, professores, parentes e redes comunitárias. Tal distanciamento social pode, no entanto, aumentar os problemas de saúde mental em crianças e adolescentes, resultando em um maior sentimento de solidão devido seus contatos sociais usuais serem restringidos pelas medidas de contenção da COVID-19 (Loades et al. 2020; Sousa et al., 2020; World Health Organization [WHO], 2020).

Dentre os impactos das medidas de distanciamento social observadas no comportamento infantil, pode-se citar a dificuldade de concentração, alteração no padrão do sono e da alimentação, maior apego aos pais ou aos responsáveis, irritabilidade, medo, solidão, tédio e maior tempo de exposição às telas (Mata, Dias, Saldanha, \& Picanço, 2020).

O distanciamento social e a hospitalização da criança ou de seus familiares são condições que podem gerar altos níveis de estresse, da mesma forma que o acesso limitado a serviços de saúde mental durante esse período pode agravar problemas psicológicos anteriores ou levar a novas dificuldades. O estresse emocional tem consequências neurobiológicas que aumentam a probabilidade de agravar doenças concomitantes e a vulnerabilidade para atender aos critérios de um transtorno mental, como transtornos de ansiedade, depressão, transtornos do sono e estresse agudo, entre outros. (Lavigne-Cerván et al. 2021).

Sendo assim, diante da relevância da temática, já que a COVID-19 é uma doença da atualidade que impactou a sociedade de diferentes formas, o presente estudo tem como objetivo realizar um levantamento na literatura científica dos impactos da COVID-19 na saúde mental de crianças.

\section{Metodologia}

Trata-se de uma revisão integrativa da literatura que inclui a síntese de vários estudos publicados, realizando-se seis etapas para sua construção: elaboração da pergunta norteadora, busca na literatura, coleta de dados, análise crítica dos estudos incluídos, discussão dos resultados e a apresentação da revisão integrativa (Ercole, Melo, \& Alcoforado, 2014; Mendes, Silveira \& Galvão, 2008; Souza, Silva, \& Carvalho, 2010).

O objetivo do estudo é responder à questão de pesquisa: "quais os impactos da COVID-19 na saúde mental das crianças durante o período da pandemia?". Com o intuito da construção da pergunta para questão de pesquisa, utilizou-se da estratégia PICO (Santos, Pimenta, \& Nobre, 2007): "P” (população) crianças; "I" (intervenção) impactos da COVID-19 na saúde mental; "C" (comparação) não se aplica, por não ser um estudo comparativo; "O" (desfecho) complicações da COVID19 na saúde mental da criança.

O levantamento dos dados ocorreu entre os meses de julho a agosto de 2021. As bases de dados pesquisadas foram: Embase, Scopus, PubMed e MEDLINE.

Os critérios de inclusão para a seleção dos estudos foram: artigos de pesquisas originais, respondesse ao objetivo proposto, open access, publicados durante o intervalo de 2020 a 2021 por serem os anos da pandemia causada pela COVID-19, até o momento. Para os critérios de exclusão adotaram-se: estudos como relatos de caso, reflexões, recomendações, revisões e literatura cinzenta (trabalho de conclusão de curso, teses, dissertações e resumos publicados em anais).

Utilizaram-se, como descritores, identificados nos Descritores em Ciência da Saúde (DECs) e seus equivalentes no Medical Subject Headings (MESH) e Embase subject headings (Emtree): "Coronavirus Infections", "COVID-19", "Child Health", "infant health", "Child, Preschool", "preschool child" e "Mental Health". A estratégia de busca foi mediante o cruzamento dos descritores por meio do operador booleano AND e OR: ("Coronavirus Infections" OR "COVID-19") AND ("Child Health" OR "infant health") AND ("Child, Preschool" OR "preschool child") AND ("Mental Health") 
De forma a sistematizar a coleta da amostra, foi utilizado o formulário de busca avançada, de acordo com cada peculiaridade de cada base de dados. A estratégia de busca nas bases de dados seguiu o protocolo apresentado no Quadro 1.

Quadro 1 - Estratégia de busca nas bases de dados. Santa Cruz, Rio Grande do Norte, Brasil, 2021.

\begin{tabular}{|l|l|c|}
\hline \multicolumn{1}{|c|}{$\begin{array}{c}\text { Base de } \\
\text { dados }\end{array}$} & \multicolumn{1}{|c|}{ Estratégia de busca } & $\begin{array}{c}\text { Resultado } \\
\text { dos } \\
\text { estudos }\end{array}$ \\
\hline $\begin{array}{l}\text { PubMed } \\
\text { Central: } \\
\text { PMC }\end{array}$ & $\begin{array}{l}\text { ("Coronavirus Infections" OR “COVID-19“) AND ("Child Health" OR “infant health") } \\
\text { AND ("Child, Preschool" OR "preschool child") AND ("Mental Health") }\end{array}$ & 81 \\
\hline SCOPUS & $\begin{array}{l}\text { "Nipple trauma" AND ("Postpartum Period" OR "Puerperium") AND ("Weaning” OR } \\
\text { "Breast Feeding") }\end{array}$ & 167 \\
\hline MEDLINE & $\begin{array}{l}\text { ("Infecções por Coronavirus" [Todos os Campos] OU "COVID-19" [Todos os Campos]) E } \\
\text { ("Saúde Infantil" [Todos os Campos] OU "Saúde infantil" [Todos os Campos]) E ("pré- } \\
\text { escola infantil" [Todos os Campos] OR "pré-escolar" [All Fields]) AND ("mental health" } \\
\text { [MeSH Terms] OR ("mental" [All Fields] AND "health" [All Fields]) OR "mental health" } \\
\text { [All Fields]) }\end{array}$ & 24 \\
\hline $\begin{array}{l}\text { EMBASE } \\
\text { Elsevier) }\end{array}$ & $\begin{array}{l}\text { ('coronavirus infections' OR 'covid-19') AND ('child health' OR 'infant health') AND } \\
\text { ('child, preschool' OR 'preschool child') AND 'mental health' }\end{array}$ & 8 \\
\hline
\end{tabular}

Fonte: Autores (2021).

Após seleção dos artigos nas bases de dados, seguiu-se a recomendação do Preferred Reporting Items for Systematic Reviews and Meta-Analyses (PRISMA) (Moher et al., 2015) conforme apresenta a Figura 1.

Figura 1 - Fluxograma de seleção dos estudos recomendação PRISMA.

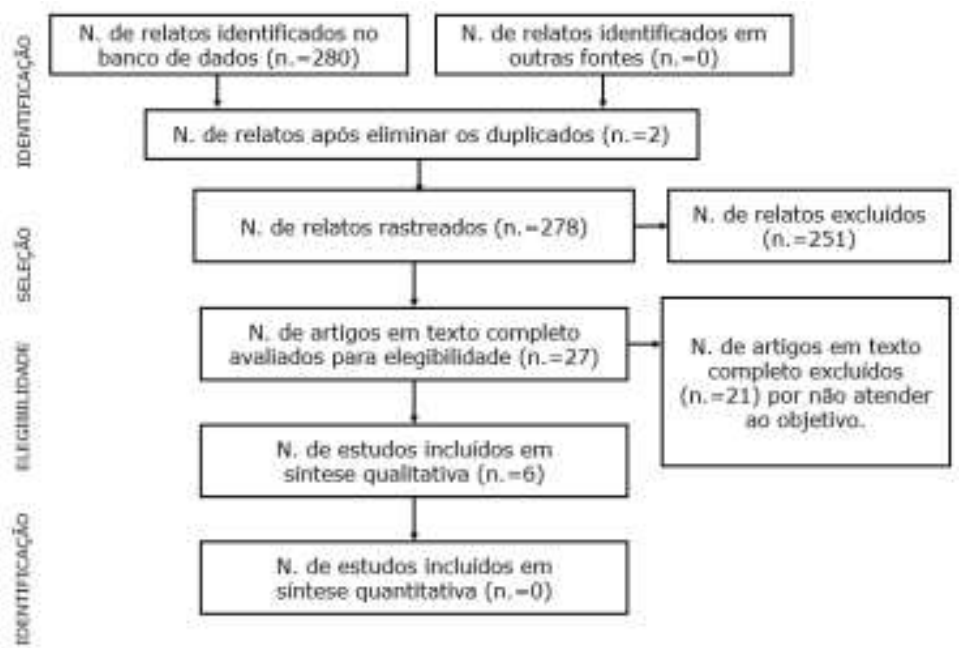

Fonte: Adaptado de Moher, Liberati, Tetzlaff e Altman (2020).

Realizou-se a leitura dos títulos e dos resumos, selecionando-os após a leitura na íntegra. Os artigos foram classificados de acordo com seu nível de evidência: Revisão sistemática ou metanálise de ensaios clínicos controlados e randomizados (nível I); ensaio clínico controlado e randomizado com intervalo de confiança estreito (nível II); resultados terapêuticos e ensaios clínicos não randomizados (nível III); estudos de caso-controle e estudos de coorte (nível IV); revisão sistemática de estudos qualitativos, descritivos e filosóficos (nível V); estudo individual de caráter descritivo e qualitativo (nível VI); e opiniões de comitês de especialistas e autoridades (nível VIII) (Stetler et al., 1998). 


\section{Resultados}

A partir da análise dos seis artigos selecionados para avaliação, dois estudos retem-se no período de 2020 e cinco estudos no período de 2021. Enquanto ao nível de evidência, os seis estudos apontam nível V. A tabela I exibe a síntese dos artigos avaliados.

Tabela 1 - Artigos disposto de acordo com a autoria, revista/ano, objetivo e método/nível de evidência. Santa Cruz, Rio Grande do Norte, Brasil, 2021.

\begin{tabular}{|c|c|c|c|c|}
\hline AUTOR & REVISTA/ ANO & OBJETIVO & MÉTODO/ NÍVEL DE EVIDÊNC & \\
\hline $\begin{array}{l}\text { Schnaiderman et } \\
\text { al. (2021) }\end{array}$ & $\begin{array}{lll}\text { Archivos Argentinos de } \\
\text { Pediatria }\end{array}$ & $\begin{array}{l}\text { Avaliar o impacto do isolamento } \\
\text { COVID-19 na saúde emocional dos } \\
\text { jovens na escola primária ou secundária. }\end{array}$ & $\begin{array}{l}\text { Estudo prospectivo, descritivo } \\
\text { transversal/V }\end{array}$ & \\
\hline $\begin{array}{l}\text { Lavigne-Cerván et } \\
\text { al. (2021) }\end{array}$ & Frontiers In Psychology & $\begin{array}{l}\text { Estudar as consequências do } \\
\text { confinamento na ansiedade, nas rotinas } \\
\text { do sono e no funcionamento executivo } \\
\text { de } 1.028 \text { crianças e adolescentes, com } \\
\text { idades entre } 6 \text { e } 18 \text { anos, residentes na } \\
\text { Espanha a; avaliar se existem diferenças } \\
\text { quanto a essas consequências em termos } \\
\text { de sexo e idade; como a ansiedade afeta } \\
\text { o funcionamento executivo em homens } \\
\text { e mulheres; e examinar as possíveis } \\
\text { correlações entre as variáveis medidas. }\end{array}$ & $\begin{array}{l}\text { Desenho associativo-comparativo } \\
\text { explicativo/V }\end{array}$ & \\
\hline $\begin{array}{l}\text { Vasileva, Alisic e } \\
\text { Young (2021) }\end{array}$ & $\begin{array}{l}\text { European Journal Of } \\
\text { Psychotraumatology }\end{array}$ & $\begin{array}{l}\text { Explorar pensamentos negativos e } \\
\text { preocupações que crianças em idade } \\
\text { pré-escolar estão tendo durante a } \\
\text { pandemia de COVID-19. }\end{array}$ & Estudo de método misto/V & \\
\hline $\begin{array}{l}\text { Mondragon, } \\
\text { Sancho, } \\
\text { Santamaria e } \\
\text { Munitis (2020) }\end{array}$ & Psychology \& Health & $\begin{array}{l}\text { Reunir as vozes das crianças em } \\
\text { confinamento durante a pandemia } \\
\text { COVID-19 na Espanha, a fim de } \\
\text { examinar como estão lidando com esta } \\
\text { crise de saúde. }\end{array}$ & Pesquisa qualitativa/V & \\
\hline $\begin{array}{l}\text { Cusinato et al. } \\
(2020)\end{array}$ & $\begin{array}{l}\text { Internacional Journal of } \\
\text { Environmental Research } \\
\text { and Public Health }\end{array}$ & $\begin{array}{l}\text { Analisar o risco potencial e os fatores de } \\
\text { proteção para o bem-estar de pais e } \\
\text { filhos durante um evento } \\
\text { potencialmente traumático, como a } \\
\text { quarentena de COVID-19. }\end{array}$ & Estudo de método misto/V & \\
\hline Masi et al. (2021) & $\begin{array}{l}\text { Journal Of Paediatrics } \\
\text { And Child Health }\end{array}$ & $\begin{array}{l}\text { Examinar o impacto da pandemia de } \\
\text { COVID-19 na saúde mental infantil e no } \\
\text { bem-estar físico e socioemocional e do } \\
\text { prestador de cuidados de saúde mental e } \\
\text { bem-estar, apoio social e uso de } \\
\text { serviços. }\end{array}$ & Estudo de método misto/V & \\
\hline
\end{tabular}




\section{Discussão}

Todos os artigos selecionados encontraram diferentes impactos negativos que a pandemia da COVID-19 causou na saúde mental de crianças. Tais impactos são causados tanto devido à mudança de rotina quanto a intensa preocupação de que eles ou seus familiares sejam infectados (Schnaiderman et al., 2021; Vasileva et al., 2021; Mondragon et al., 2020). Além disso, os efeitos da pandemia na vida dos próprios pais também foram responsáveis por afetar negativamente a saúde mental das crianças (Cusinato et al., 2020).

Ao avaliar o impacto psicológico do isolamento social em jovens de São Carlos de Bariloche, na Argentina, o estudo de Schnaiderman et al. (2021) constatou que 96,3\% dos pais que participaram do estudo verificaram mudanças emocionais e comportamentais em seus filhos. As mais relatadas foram que estavam mais entediados, irritáveis, apáticos e zangados. Modificações na rotina podem ter sido a causa do aumento de tais emoções, como o fato de ir dormir e acordar mais tarde e maior tempo de uso de telas para entretenimento.

O estudo de Vasileva et al. (2021), que explorou a presença de pensamentos negativos e preocupações em crianças em idade pré-escolar durante a pandemia de COVID-19, também encontrou resultados semelhantes. Além da dificuldade em compreender a causalidade e superestimar o risco de contrair a doença, os cuidadores relataram que as crianças apresentavam preocupação em ficar doente, infectar outras pessoas e de que a rotina nunca mais voltasse ao normal. Também foram relatadas mudanças comportamentais como maior inquietação, cautela, evitação e busca de apego.

O estudo de Mondragon et al. (2020) avaliou como crianças da Espanha estão lidando com pandemia causada pela COVID-19. Diferentemente dos outros já mencionados, os pesquisadores desse estudo não ouviram relatos dos responsáveis, mas sim das próprias crianças e constataram emoções confusas e sentimentos negativos como medo, nervosismo, preocupação, solidão, tristeza, tédio e raiva. Além disso, as crianças também relataram sentir falta de colegas, familiares e de fazer exercícios ao ar livre.

Assim sendo, as medidas de distanciamento social também deixaram as crianças mais sedentárias. Masi et al. (2021) em seu estudo que avaliou o impacto da pandemia COVID-19 no bem-estar de crianças com deficiência de desenvolvimento neurológico e seus pais, observou que durante a pandemia as crianças passaram a assistir mais à televisão e às mídias digitais, fazer menos exercícios, ter uma pior qualidade do sono e se alimentar pior. Em consequência disso, a maioria dos pais participantes do estudo relataram que a saúde e o bem-estar de seus filhos foram afetados pela COVID-19.

Outro fator que também parece ter contribuído para afetar o bem-estar das crianças durante a pandemia foram os desajustes psicológicos causados nos próprios pais. O estudo de Cusinato et al. (2020) avaliou o estresse, resiliência e bemestar em crianças italianas e seus pais durante a pandemia da COVID-19 e constatou que as medidas de confinamento, mudanças na rotina e nas condições de trabalho afetam negativamente as dimensões psicológicas dos pais e, por sua vez, o bem-estar de seus filhos.

De forma semelhante, Lavigne-Cerván et al. (2021), analisaram as consequências do confinamento na ansiedade, nas rotinas do sono e no funcionamento executivo de 1.028 crianças e adolescentes, de 6 a 18 anos, residentes na Espanha. Os resultados mostraram que a situação de confinamento devido à pandemia pode estar aumentando os níveis de ansiedade na população estudada, o que, consequentemente, pode afetar o sono e impactar negativamente suas ações, pensamentos e emoções.

Referente a limitação do estudo foi o baixo número de artigos identificados até o presente momento na literatura científica, justificado pela limitação de estudos científicos em relação ao tema e a população, interferindo em um compilado mais abrangente dos dados. Apesar das limitações, foi possível realizar a discussão sobre o objetivo da revisão. 


\section{Conclusão}

Mediante o exposto, em relação à saúde das crianças em tempos de pandemia, é possível afirmar que a COVID-19 trouxe diversas implicações negativas, principalmente impactos relacionados à saúde mental causados pelo isolamento social. Além de não estarem acostumadas a viverem durante muito tempo em isolamento social, as mudanças na rotina, a intensa preocupação de serem infectadas e até os problemas dos próprios pais, acarretam em distúrbios em suas ações, pensamentos e emoções, o que pode gerar transtornos mais graves como níveis de ansiedade aumentados, depressão ou até mesmo agravar problemas mentais já existentes.

Nesse contexto, é fundamental a adoção de medidas para mantê-las saudáveis, como a prática de atividades físicas que possam ser realizadas dentro de suas próprias casas, brincadeiras que não envolvam a utilização de telas e a realização de refeições em família.

Dessa forma, torna-se importante a necessidade de novos estudos referentes ao tema, como estudos observacionais do tipo analítico, com o objetivo de entender o desfecho relacionado a saúde mental das crianças no período pandêmico e contribuir cientificamente com o conhecimento para a prática dos profissionais e a comunidade acadêmica.

\section{Referências}

Campos, M. R., Schramm, J. M. A., Emmerick, I. C. M., Rodrigues, J. M., de Avelar, F. G., \& Pimentel, T. G. (2020). Carga de doença da COVID-19 e de suas complicações agudas e crônicas: reflexões sobre a mensuração (DALY) e perspectivas no Sistema Único de Saúde. Cadernos de Saúde Pública, 36(11), 1-14. https://doi.org/10.1590/0102-311x00148920

Cusinato, M., Iannattone, S., Spoto, A., Poli, M., Moretti, C., Gatta, M., \& Miscioscia, M. (2020). Stress, Resilience, and Well-Being in Italian Children and Their Parents during the COVID-19 Pandemic. International Journal of Environmental Research and Public Health, 17(22), 82-97. https://doi.org/10.3390/ijerph17228297

Ercole, F. F., Melo, L. S., \& Alcoforado, C. L. G. C. (2014). Revisão Integrativa versus Revisão Sistemática. Revista Mineira de Enfermagem, 18(1), 1-3. http://dx.doi.org/10.5935/1415-2762.20140001

Guan, W., Ni, Z., Yu, H., Liang, W., Ou, C., He, J., Liu, L., Shan, H., Lei, C., Hui, D. S. C., Du, B., Li, L., Zeng, G., Yuen, K., Chen, R., Tang, C., Wang, T., Chen, P., Xiang, J., Li, S., Wang, J., Liang, Z., Peng, Y., Wei, L., Liu, Y., Hu, Y., Peng, P., Wang, J., Liu, J., Chen, Z., Li, G., Zheng, Z., Qiu, S., Luo, J., Ye, C., Zhu, S., \& Zhong, N. (2020). Clinical Characteristics of Coronavirus Disease 2019 in China. New England journal of medicine, 382(18), 1708-1720. https://doi.org/10.1056/nejmoa2002032

Henrique, A., Gonçalves, C., César, V., \& Candeia, U. (2020). Frequência de crianças com o novo coronavírus: revisão sistemática. Comunicação em Ciências da Saúde, 31(3), 89-100. https://doi.org/10.51723/ccs.v31i03.750

Lavigne-Cerván, R., Costa-López, B., de Meir, R. J., Real-Fernández, M., de León, M. S., \& Navarro-Soria, I. (2021). Consequences of COVID-19 Confinement on Anxiety, Sleep and Executive Functions of Children and Adolescents in Spain. Frontiers in Psychology, 12(1), 1-11. https://doi.org/10.3389/fpsyg.2021.565516

Loades, M. E., Chatburn, E., Higson-Sweeney, N., Reynolds, S., Shafran, R., Brigden, A., Linney, C., McManus, M. N., Borwick, C., \& Crawley, E. (2020). Rapid Systematic Review: The Impact of Social Isolation and Loneliness on the Mental Health of Children and Adolescents in the Context of COVID-19. Journal of the American Academy of Child \& Adolescent Psychiatry, 59(11), 1218-1239. https://doi.org/10.1016/j.jaac.2020.05.009

Masi, A., Diaz, A. M., Tully, L., Azim, S. I., Woolfenden, S., Efron, D., \& Eapen, V. (2020). Impact of the COVID-19 pandemic on the well-being of children with neurodevelopmental disabilities and their parents. Journal of Pediatrics and Child Health, 1-6. https://doi.org/10.1111/jpc.15285

Mata, I. R. S., Dias, L. S. C., Saldanha, C. T., \& Picanço, M. R. de A. (2020). As implicações da pandemia da COVID-19 na saúde mental e no comportamento das crianças. Residência Pediátrica, 10(3), 1-5. https://doi.org/10.25060/residpediatr

Mendes, K. S., Silveira, R. C. C. P., \& Galvão, C. M. (2008). Revisão integrativa: método de pesquisa para a incorporação de evidências na saúde e na enfermagem. Texto \& Contexto - Enfermagem, 17(4), 758-764. http://dx.doi.org/10.1590/s0104-07072008000400018

Moher, D., Shamseer, L., Clarke, M., Ghersi, D., Liberati, A., Petticrew, M., Shekelle, P., Stewart, L. A., \& Group P. (2015). Preferred reporting items for systematic review and meta-analysis protocols (PRISMA-P) 2015 statement. Systematic Reviews, 4(1), 1-9. 10.1186/2046-4053-4-1

Mondragon, N. I., Sancho, N. B., Santamaria, M. D., \& Munitis, A. E. (2020). Struggling to breathe: a qualitative study of children's wellbeing during lockdown in Spain. Psychology \& Health, 36(2), 179-194. https://doi.org/10.1080/08870446.2020.1804570

Rocha, M. F. A., Veloso, W. G., Bezerra, R. E. A., Gomes, L. A., \& Marcolino, A. B. L. (2021). O impacto da pandemia do covid-19 na saúde infanto-juvenil: um estudo transversal. Brazilian Journal of Health Review, 4(1), 3483-3497. https://doi.org/10.34119/bjhrv4n1-271 
Research, Society and Development, v. 10, n. 15, e115101522448, 2021

(CC BY 4.0) | ISSN 2525-3409 | DOI: http://dx.doi.org/10.33448/rsd-v10i15.22448

Sant'Ana, G., Imoto, A. M., Amorim, F. F., Taminato, M., Peccin, M. S., Santana, L. A., Göttems, L. B. D., \& Camargo, E. B. (2020). Infecção e óbitos de profissionais da saúde por COVID-19: revisão sistemática. Acta Paulista de Enfermagem, 33, 1-9. https://doi.org/10.37689/actaape/2020AO0107ape/2020ao0107

Santos, C. M. C., Pimenta, C. A. M., \& Nobre, M. R. C. (2007). The PICO strategy for the research question construction and evidence search. Revista LatinoAmericana de Enfermagem, 15(3), 508-511. http://dx.doi.org/10.1590/s0104-11692007000300023

Schnaiderman, D., Bailac, M., Borak, L., Comar, H., Eisner, A., Ferrari, A., Giannini, G., Risso, F., Vetere, C., \& Garibotti, G. (2021). Impacto psicológico del aislamiento por COVID-19 en jóvenes de San Carlos de Bariloche, Argentina: la mirada de los padres. Archivos Argentinos de Pediatria, 119(3), 1-7. http://dx.doi.org/10.5546/aap.2021.170

Sousa, G. O., Sales, B. N., Rodrigues, A. M. X., Rocha, G. M. M., \& de Oliveira, G. A. L. (2020). Evolução epidemiológica da COVID-19 no Brasil e no mundo. Research, Society and Development, 9(7), 1-13. https://doi.org/10.33448/rsd-v9i7.4653

Souza, M. T., Silva, M. D., \& Carvalho, R. (2010). Integrative review: what is it? how to do it? Einstein (São Paulo), 8(1), 102-106. http://dx.doi.org/10.1590/s1679-45082010rw1134

Stetler, F. C. B., Morsi, D., Rucki, S., Broughton, S., Corrigan, B., Fitzgerald, C. J., Giuliano, K., Havener, P., \& Sheridan, A. (1998). Utilization-focused integrative reviews in a nursing service. Applied Nursing Research, 11(4), 195-206. http://dx.doi.org/10.1016/s0897-1897(98)80329-7

Vasileva, M., Alisic, E., \& Young, A. (2021). Covid-19 desenmascarado: pensamientos negativos y preocupaciones de niños preescolares durante la pandemia de covid-19 en Australia. European journal of psychotraumatology, $12(1), \quad 1-11 . \quad$ https://doiorg.ez18.periodicos.capes.gov.br/10.1080/20008198.2021.1924442

Wang, C., Horby, P. W., Hayden, F. G., \& Gao, G. F. (2020). A novel coronavirus outbreak of global health concern. The Lancet, 395(10223), 470-473. https://doi.org/10.1016/S0140-6736(20)30185-9

World Health Organization. (2020). Breastfeeding and COVID-19. WHO. https://www.who.int/news-room/commentaries/detail/breastfeeding-and-covid-19 